\title{
Steroid myopathy in a child with diffuse alveolar hemorrhage: a case report
}

\author{
Yongshuang Fang^, Lang Yuan, Ran Zhao, Kun Jiang, Xiaoyan Dong \\ Department of Respiratory Medicine, Children's Hospital of Shanghai, Shanghai Jiao Tong University, Shanghai, China \\ Correspondence to: Xiaoyan Dong, MD. Chief physician. No. 355 Luding Road, Shanghai 200062, China. Email: dongxy1515@126.com.
}

\begin{abstract}
Steroid myopathy (SM) is a side effect of glucocorticoid administration. Previous reports of SM after glucocorticoid therapy for respiratory diseases have focused on asthma and chronic obstructive pulmonary disease in adults or adolescents. To our knowledge, there have been no reports of SM after treatment with glucocorticoid for diffuse alveolar hemorrhage (DAH) in preschoolers. We present a 4-year-old girl with DAH requiring the treatment with methylprednisolone, who was transferred to the pediatric intensive care unit (PICU) due to respiratory failure caused by severe pneumonia requiring mechanical ventilation. When transferred to respiratory department, the strength of the limbs of the patient decreased, and her lower limbs were paralyzed. We performed relevant examination to rule out juvenile dermatomyositis and other diseases such as deep venous thrombosis of lower limbs, Guillain-Barre syndrome that may cause the decline of muscle strength. SM was considered based on the clinical characteristics and risk factors of the child, so glucocorticoid treatment was tapered following the treatment regimen for DAH and SM. The muscle strength of the child completely recovered, which further confirmed our diagnosis of SM. In pediatrics, SM should also be a concern when patients with respiratory disease experience decreased muscle strength following glucocorticoid therapy.
\end{abstract}

Keywords: Diffuse alveolar hemorrhage (DAH); child; steroid myopathy (SM); case report

Submitted Apr 06, 2021. Accepted for publication Apr 28, 2021.

doi: 10.21037/apm-21-816

View this article at: http://dx.doi.org/10.21037/apm-21-816

\section{Introduction}

Steroid myopathy (SM) is a non-inflammatory toxic myopathy caused by either endogenous or exogenous excess of steroid, which is mainly characterized by muscle weakness and muscle atrophy in the proximal extremities (1), which is a common complication of diseases where glucocorticoids are the main treatment (2). For respiratory diseases, previous reports of SM after glucocorticoid therapy have focused on asthma and chronic obstructive pulmonary disease in adults or adolescents $(3,4)$. However, SM rarely occurs in young children with respiratory disease. To our knowledge, there have been no reports of SM after treatment with glucocorticoid for diffuse alveolar hemorrhage (DAH) in preschoolers. In this paper, we report a case of DAH appearing chronic SM by receiving treatment of glucocorticoids in a 4-year-old girl and review the clinical features, diagnosis, treatment and prognosis of SM.

We present the following article in accordance with the CARE reporting checklist (available at http://dx.doi. org/10.21037/apm-21-816).

\section{Case presentation}

All procedures performed in studies involving human participants were in accordance with the ethical standards of the institutional and national research committee

\footnotetext{
^ ORCID: 0000-0002-1247-4209.
} 


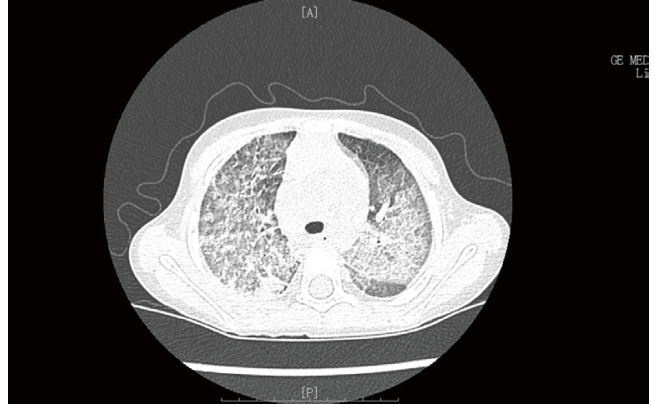

Figure 1 Diffuse bilateral ground-glass opacification of lungs.

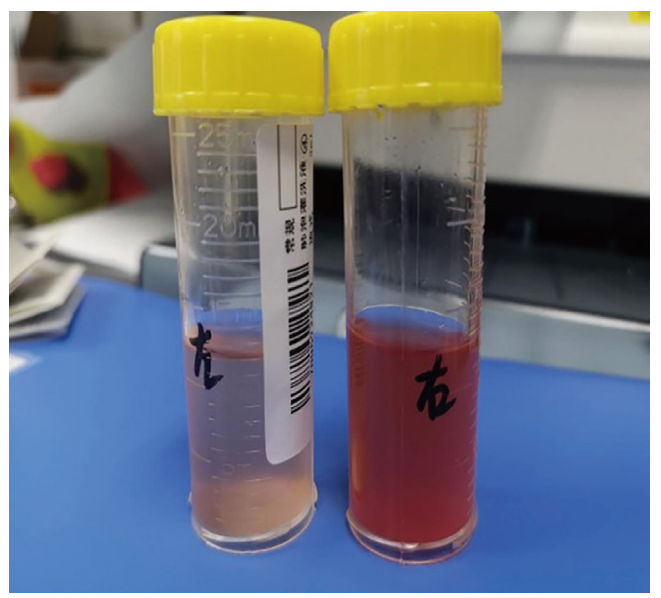

Figure 2 Bloody alveolar lavage fluid.

and with the Helsinki Declaration (as revised in 2013). Written informed consent was obtained from the patient. A 4-year-old Chinese girl with unremarkable medical and developmental history was admitted to the hematology department due to recurrent anemia for more than 1 year. The girl's physical examination at that time, including assessment of growth and development, was normal except for pale complexion and hyoxemia. Blood routine examination revealed severe microcytic hypochromic anemia (RBC $2.34 \times 10^{9} / \mathrm{L}$, hemoglobin $60 \mathrm{~g} / \mathrm{L}, \mathrm{MCV} 88.5 \mathrm{fL}, \mathrm{MCH}$ $25.16 \mathrm{pg}$ ) with a reticulocyte count of $12.91 \%$. Osmotic RBC fragility, Glucose-6-phosphate dehydrogenase were normal. The anti-human globulin test was negative. Indirect bilirubin level was within the normal range. Blood routine examination of both parents was normal, as well. Bone marrow aspiration was normal. Related laboratory tests did not support hemolytic or hematopoietic disorders. During hospitalization, the child had fever, cough and hyoxemia. This information prompted a high-resolution computed tomography (HRCT) of the chest which showed diffuse bilateral ground-glass opacification, as shown in Figure 1. We performed a bronchoscopy and found that the alveolar lavage fluid was bloody, as shown in Figure 2. Considering the history of anemia, cough, and hypoxemia, as well as chest CT findings and bloody alveolar lavage fluid, DAH was suspected. Additional investigations were performed to rule out possible causes for alveolar bleeding. Echocardiography and right catheterization showed no abnormal cardiac anatomy and no evidence of pulmonary hypertension. The mean pulmonary artery pressure (mPAP) measured by right catheterization was $18 \mathrm{mmHg}$. Serum antibodies to antinuclear antibodies, and antineutrophil cytoplasmic antibodies (c-ANCA, p-ANCA) were negative. Goodpasture syndrome was excluded by negative antiglomerular basement membrane antibodies, normal urinalysis, and normal kidney function tests. Pathogen testing does not support bacterial, fungal, viral or other infections with specific agents. The diagnosis of DAH was confirmed, and methylprednisolone $(40 \mathrm{mg} / \mathrm{d}$, equivalents to $2 \mathrm{mg} / \mathrm{kg} / \mathrm{d}$ ) was used intravenously to treat the primary disease. The patient's symptoms of hypoxemia and anemia gradually improved and she was discharged. She continued to take methylprednisolone tablets orally $(40 \mathrm{mg} / \mathrm{d}$, equivalents to $2 \mathrm{mg} / \mathrm{kg} / \mathrm{d}$ ).

After 3 weeks of treatment with methylprednisolone ( $2 \mathrm{mg} / \mathrm{kg} / \mathrm{d})$, the patient was admitted to hospital again due to enteric adenovirus infection characterizing by diarrhea, abdominal distension, fatigue and poor appetite. On days 1 through 7 of hospitalization, in addition to oral methylprednisolone tablets, the patient received supportive treatment including intravenous fluids for maintaining water and electrolyte balance and probiotics for regulating intestinal flora. After treatment, the symptoms of her intestinal adenovirus infection gradually improved, and no adenovirus antigen was found in the second stool examination and no adenovirus antibody was found in serum antibody examination.

On the 7th hospital day during hospitalization, the child developed cough, followed by fever, chest pain, progressive breathing difficulty, skin pallor and hypoxemia. Chest CT showed exudation of the upper and lower lobes of both lungs accompanied by consolidation of the lower lobe of the right lung, as shown in Figure 3. Blood routine examination suggested high index of infection and low hemoglobin (CRP $42 \mathrm{mg} / \mathrm{L}, \mathrm{HB} 67 \mathrm{~g} / \mathrm{L}$ ). No positive pathogenic bacteria were found in respiratory tract etiology. On the 9th hospital day, she developed respiratory failure and was transferred to the 

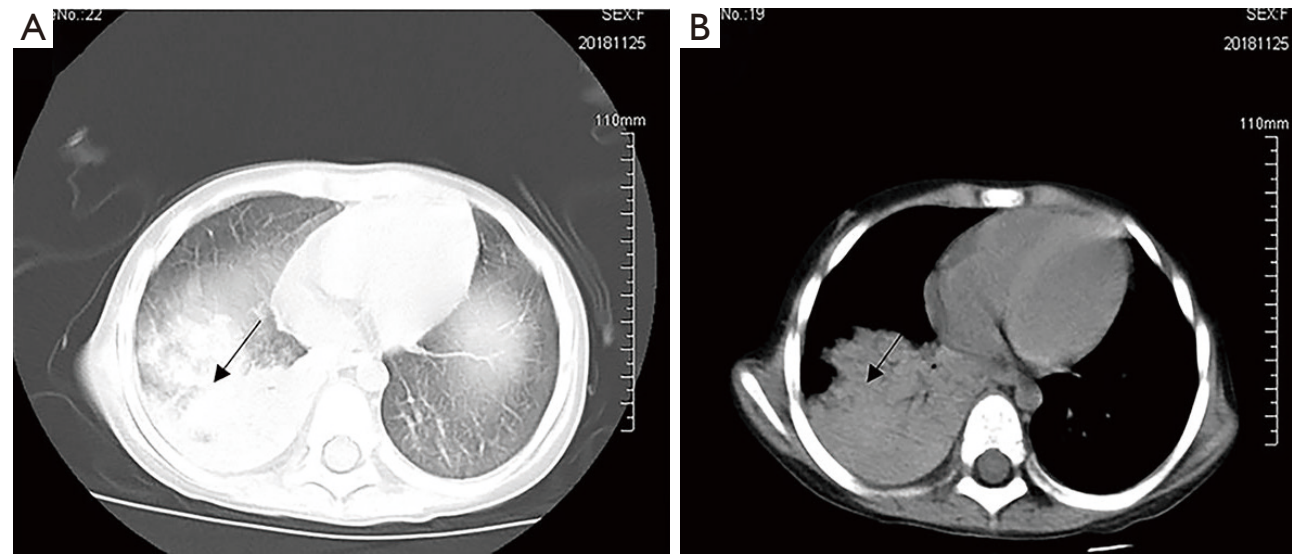

Figure 3 Exudation of the upper and lower lobes of both lungs accompanied by consolidation of the lower lobe of the right lung (black arrow). (A) The lung window. (B) The mediastinal window.
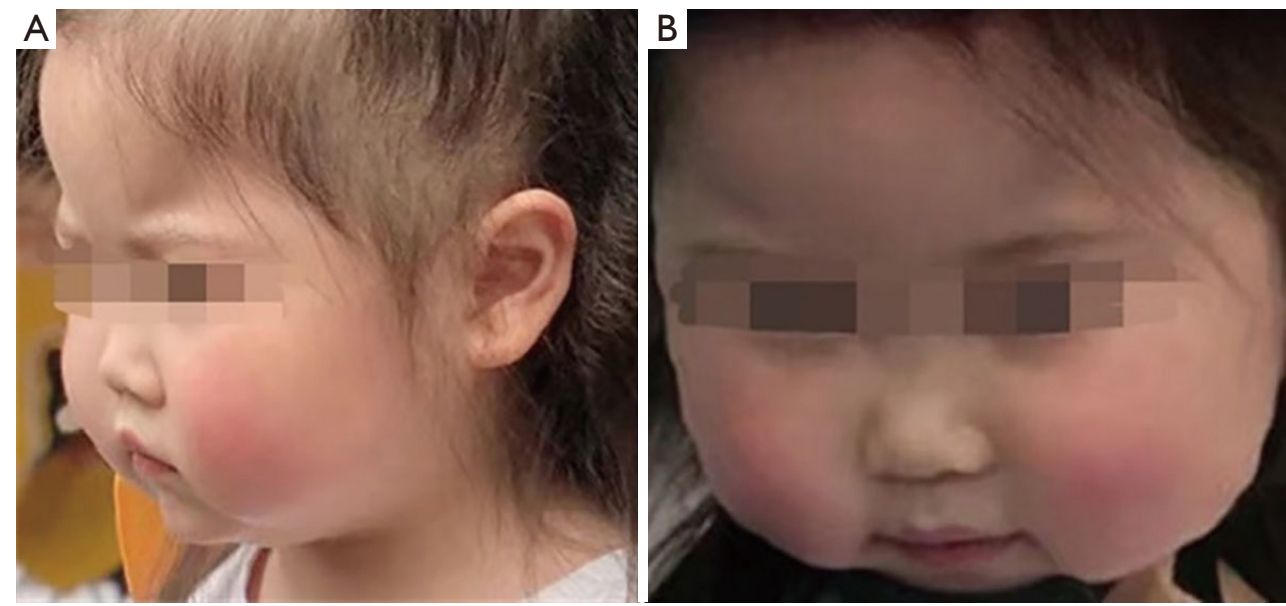

Figure 4 Cushing appearance of the patient.

pediatric intensive care unit (PICU). She was subsequently intubated and put on mechanical ventilation at the PICU. We treated the child with anti-infection and transfusion of suspended red blood cells. On the 12th hospital day (the 4th day of being transferred to PICU), she was successfully liberated from mechanical ventilation and began to receive oxygen through a nasal catheter. The next day, she was transferred to general ward of respiratory department for further treatment. Cushing appearance of the girl gradually got worse, presenting the appearance of moon face, buffalo hump, as shown in Figure 4. At the same time, muscle strength on arms was grade IV, muscle strength on legs was grade III. We conducted relevant examinations to exclude diseases such as deep venous thrombosis of lower limbs, Guillain-Barre syndrome and other diseases that may cause decreased muscle strength.

The patient presented with symmetric proximal muscle weakness and interstitial lung disease, which partly consistent with juvenile dermatomyositis (5-7). However, typical dermatomyositis also has the following manifestations (8): (I) a typical rash should present; (II) laboratory tests should suggest an elevated serum myosin level; (III) EMG should present typical triad changes; (IV) MRI findings such as muscle edema and fatty infiltration are common; (V) specific autoantibodies can be detected in the serum. In this case, no typical rash presented, at the same time, no calf muscle hypertrophy presented, and Shoulder sign was negative. Blood biochemistry test showed lactate dehydrogenase $(\mathrm{LDH})$ was increased (LDH $383 \mathrm{U} / \mathrm{L})$, creatine kinase (CK) and myocardial enzyme 

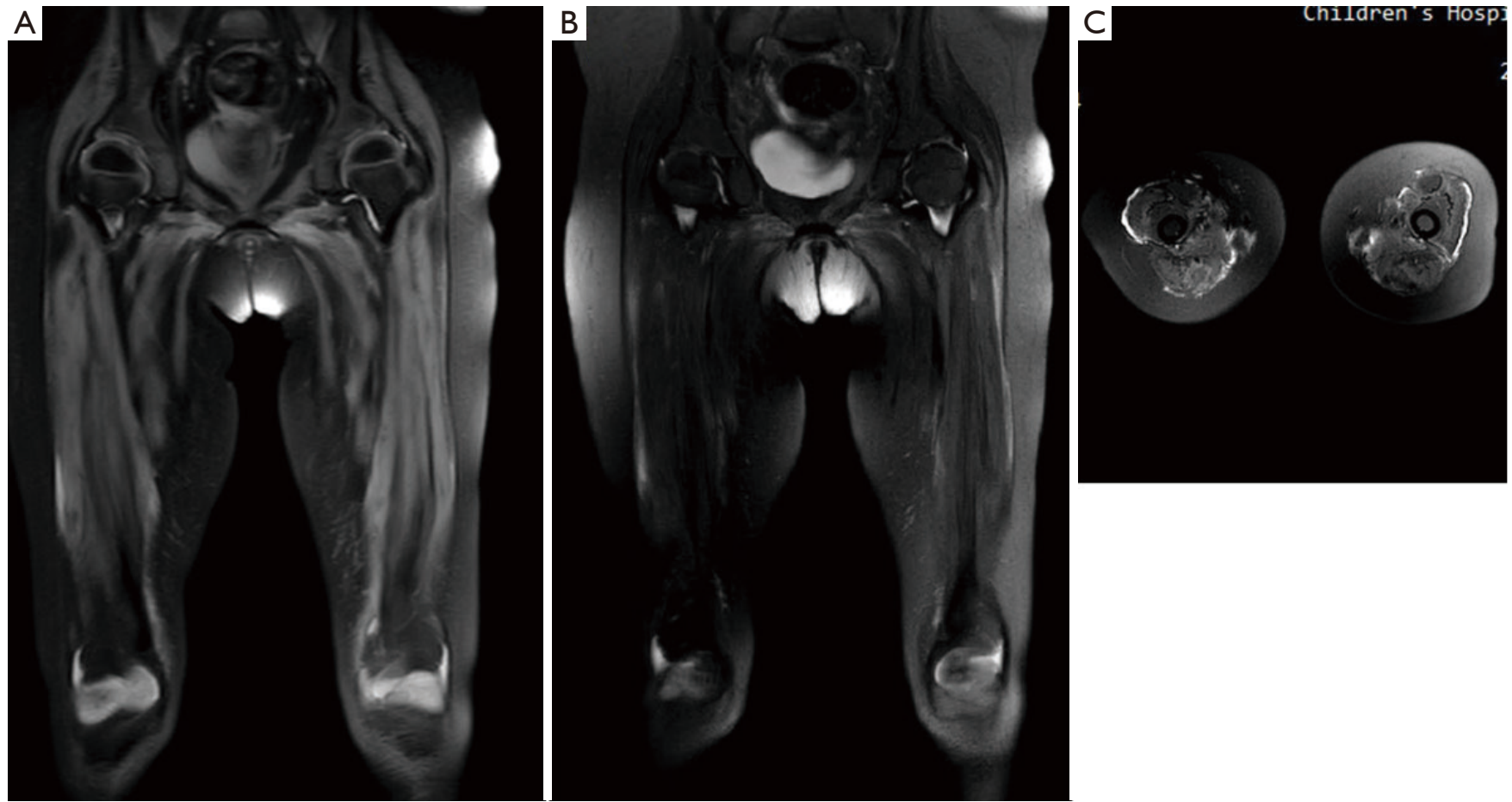

Figure 5 MRI findings on the proximal muscle area. (A) PDW SPAIR sequence; (B,C) T2W-TSE SPIR sequences.

were not increased (CK 149 U/L, CK-MB 7 U/L). The EMG showed myogenic changes but no dermatomyositis triad. Myositis autoantibody test was negative. MRI of both lower limbs showed diffuse muscle edema without fat infiltration, as shown in Figure 5. Muscle biopsy was rejected by the parents because it was invasive. The clinical manifestations and examinations did not meet the diagnostic criteria of dermatomyositis (8).

SM is one of the manifestations of endogenous Cushing's syndrome and a side effect of glucocorticoid therapy (9). The risk of acquiring a glucocorticoid-induced myopathy is higher in respiratory distress syndrome, and those who are physically inactive and in negative nitrogen balance at the onset of glucocorticoid treatment (10). In this case, the girl experienced the risks: (I) she has a long history of methylprednisolone use ( 5 weeks, $40 \mathrm{mg} / \mathrm{d}$, equivalents to $2 \mathrm{mg} / \mathrm{kg} / \mathrm{d}$ ), and her Cushing appearance was obvious. (II) She had respiratory distress syndrome and respiratory failure in the course of the disease. (III) In the early stage of the disease, she developed intestinal infection, and her activity was reduced due to general fatigue. Considering that the patient had a history of glucocorticoid use and related risk factors that she experienced, we were inclined to the diagnose of SM. Therefore, the dosage of methylprednisolone we gradually reduced in accordance with the treatment plan for DAH, which was also consistent with the treatment principles of SM. The dosage of methylprednisolone was reduced $4 \mathrm{mg}$ every 1 to 2 weeks. When the dosage was reduced to $12 \mathrm{mg} / \mathrm{d}$ (equal to $0.6 \mathrm{mg} / \mathrm{kg} / \mathrm{d}$ ), we slowed down the reduction of methylprednisolone. The dosage of methylprednisolone is shown in Figure 6.

Outpatient follow-up showed that during the reduction of the dose of methylprednisolone, the patient did not develop anemia, her oxygen demand gradually decreased, and muscle strength gradually recovered. When the dosage was reduced to $8 \mathrm{mg} / \mathrm{d}(0.4 \mathrm{mg} / \mathrm{kg} / \mathrm{d})$, the muscle strength returned to normal, which confirmed our diagnosis of SM. The timeline of the case was demonstrated in Figure 7.

\section{Discussion}

$\mathrm{DAH}$ is a clinical syndrome manifested range from lifethreatening acute respiratory failure to hidden symptoms such as cough. In most symptomatic cases, hemoptysis, dyspnea, hypoxemia, and chest radiography alveolar infiltration are present. Because children won't cough up phlegm, hemoptysis may not appear. Anemia is one 


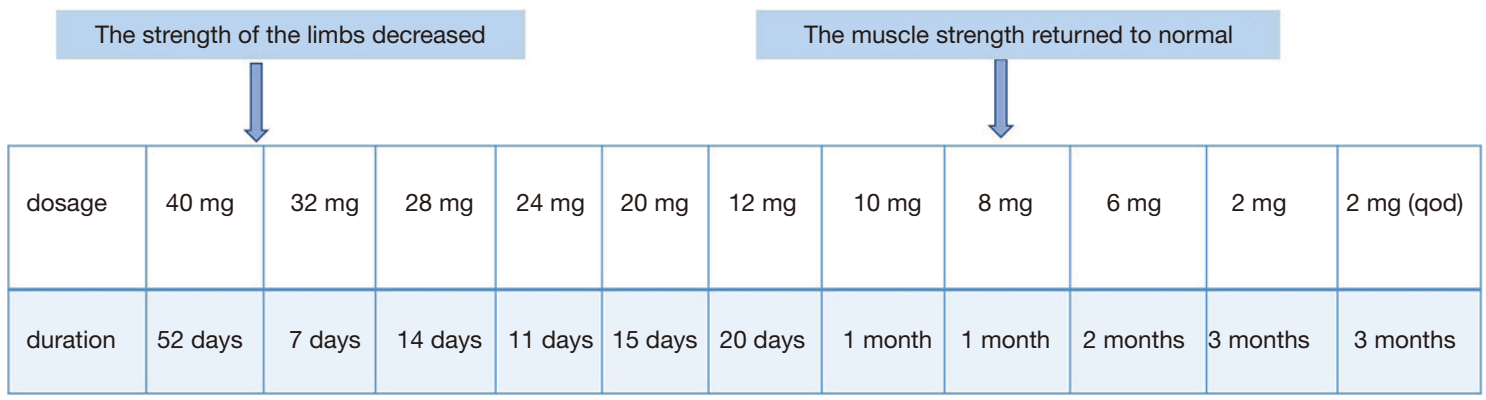

Figure 6 Dosage of methylprednisolone in the course of disease.

of the important signs in the diagnosis of DAH $(11,12)$. The treatment of DAH is empirical and ranges from supportive treatment to high-dose corticosteroids, immunosuppressants, and plasmapheresis (13).

$\mathrm{SM}$ is a non-inflammatory toxic myopathy caused by either endogenous or exogenous excess of steroid, which is mainly manifested by muscle weakness and muscle atrophy in the proximal extremities (1). Clinical manifestations include: (I) muscle wasting, weakness, and fatigability (1); (II) symptoms of Cushing's syndrome, such as diabetes mellitus, mood disorders, fragile skin and osteoporosis, are often observed but not always present (14).

The risk of acquiring a SM are as follows: (I) all patients have a history of glucocorticoid use. The dosage of glucocorticoid that can cause myopathy varies widely among patients (10). There have been reports of acute SM with small doses of steroid hormones at home and abroad $(15,16)$, especially for fluorinated steroid compounds which are more likely to cause SM than non-fluorinated formulations $(1,10)$; (II) a history of hospitalization in intensive care unit (ICU); (III) the elderly, patients with cancer, respiratory distress syndrome, and those who are physically inactive and in negative nitrogen balance at the onset of glucocorticoid treatment (10).

The diagnosis of SM is a clinical diagnosis. At present, there are various methods of auxiliary examination for SM. Muscle biopsy reveals non-specific type II fiber atrophy and a significant lack of necrosis or regeneration $(1,17,18)$. In most SM patients, needle electromyography (EMG) is normal. Only in few patients, EMG reveals a mild reduction of the amplitude of the motor unit action potentials with low-grade spontaneous activity (18). Magnetic resonance imaging (MRI) is another recent tool for the investigation of myopathy, but no study has been conducted to investigate the usefulness of MRI for diagnosis and monitoring of SM up to now. Laboratory tests may show elevated levels of serum CK, as well as significant increases in urinary creatine and 3-methylhistidine, but no reliable biomarker that could be utilized in clinical and research settings to identify SM, track its progression, and monitor its response to interventions is available currently (9).

In patients with SM, the utilization of glucocorticoids should be discontinued or reduced, except the clinical status of the patient contra-indicates such a change (10).

The clinical manifestations and laboratory tests of our patient were partly similar to dermatomyositis, but mostly consistent with the characteristics of SM. In clinical practice, the differentiation of these two diseases is very important and directly related to the prognosis of patients (10), so we reviewed the relevant literature for differentiation, as is shown in Table 1.

According to the differential points indicated in the table, combined with the clinical manifestations, relevant laboratory tests and related risk factors, we finally diagnosed $\mathrm{SM}$, and the strength of the child returned to normal after glucocorticoid reduction, which further confirmed our diagnosis.

We report a case of chronic SM after glucocorticoid treatment in a child with DAH. Pediatric respiratory diseases, such as interstitial lung disease and asthma, require glucocorticoid therapy. If patients experience decreased muscle strength during treatment, it is important to be careful to differentiate between the primary disease or SM after treatment with glucocorticoid. When SM is suspected, the use of glucocorticoids should be discontinued or gradually reduced on the premise that the primary disease is well controlled. 


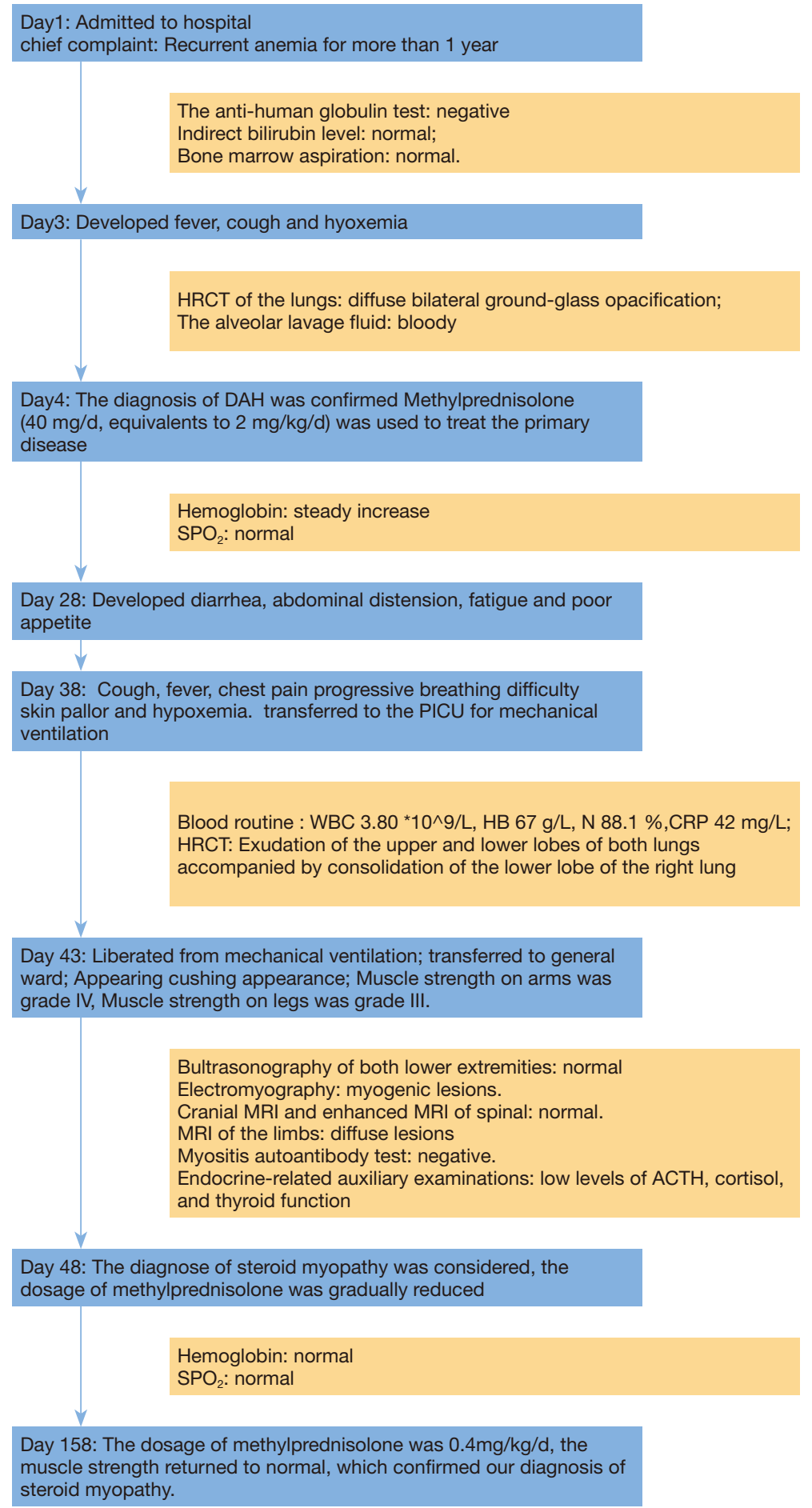

Figure 7 The timeline of the case. DAH, diffuse alveolar hemorrhage; MRI, magnetic resonance imaging; HRCT, high-resolution computed tomography; CT, computed tomography; mPAP, mean pulmonary artery pressure; PICU, pediatric intensive care unit; EMG, electromyography; ACTH, adrenocorticotropic Hormone; CK, creatine kinase; ALT, alanine aminotransferase; AST, aspartate aminotransferase; LDH, lactate dehydrogenase. 
Table 1 The differentiation of inflammatory myopathy and steroid myopathy

\begin{tabular}{|c|c|c|}
\hline & Juvenile dermatomyositis & Steroid myopathy \\
\hline $\begin{array}{l}\text { Symmetrical proximal muscle } \\
\text { weakness }\end{array}$ & Present & Present \\
\hline Cushing's syndrome & Present or absent & Present \\
\hline Muscle enzyme levels & $\begin{array}{l}\text { Muscle enzymes include CK, ALT, AST, LDH and } \\
\text { aldolase (19) }\end{array}$ & Normal or slightly elevated (10) \\
\hline Urinary creatine & $\begin{array}{l}\text { Elevated, but increases further when } \\
\text { glucocorticoid is discontinued }\end{array}$ & $\begin{array}{l}\text { Elevated, but reduces when glucocorticoid is } \\
\text { discontinued }\end{array}$ \\
\hline Electromyography (EMG) & $\begin{array}{l}\text { Fibrillation potentials and positive sharp waves } \\
\text { and Short-duration, small-amplitude, polyphasic } \\
\text { motor units and early recruitment during a } \\
\text { voluntary muscle contraction (8) }\end{array}$ & $\begin{array}{l}\text { Normal or a mild reduction of the amplitude } \\
\text { of the motor unit action potentials with low- } \\
\text { grade spontaneous activity (9) }\end{array}$ \\
\hline Muscle biopsy & Perifascicular atrophy, necrosis, degeneration & $\begin{array}{l}\text { Nonspecific atrophy of type llb muscle } \\
\text { fibers (10) }\end{array}$ \\
\hline
\end{tabular}

CK, creatine kinase; ALT, alanine aminotransferase; AST, aspartate aminotransferase; LDH, lactate dehydrogenase; MRI, magnetic resonance imaging.

\section{Acknowledgments}

Funding: This study was funded by Shanghai International Science and Technology Cooperation Fund Project, 18410721300, and Shanghai Jiao Tong University medical engineering Cross project, YG2017MS34.

\section{Footnote}

Reporting Checklist: The authors have completed the CARE reporting checklist. Available at http://dx.doi.org/10.21037/ apm-21-816

Conflicts of Interest: All authors have completed the ICMJE uniform disclosure form (available at http://dx.doi. org/10.21037/apm-21-816). The authors have no conflicts of interest to declare.

Ethical Statement: The authors are accountable for all aspects of the work in ensuring that questions related to the accuracy or integrity of any part of the work are appropriately investigated and resolved. All procedures performed in studies involving human participants were in accordance with the ethical standards of the institutional and national research committee and with the Helsinki Declaration (as revised in 2013). Written informed consent was obtained from the patient.

Open Access Statement: This is an Open Access article distributed in accordance with the Creative Commons Attribution-NonCommercial-NoDerivs 4.0 International License (CC BY-NC-ND 4.0), which permits the noncommercial replication and distribution of the article with the strict proviso that no changes or edits are made and the original work is properly cited (including links to both the formal publication through the relevant DOI and the license). See: https://creativecommons.org/licenses/by-nc$\mathrm{nd} / 4.0 /$. 


\section{References}

1. Minetto MA, Lanfranco F, Motta G, et al. Steroid myopathy: Some unresolved issues. J Endocrinol Invest 2011;34:370-5.

2. Simon NG. A new diagnostic tool for the detection of steroid myopathy. Clin Neurophysiol 2019;130:1407-8.

3. Decramer M, Stas KJ. Corticosteroid-induced myopathy involving respiratory muscles in patients with chronic obstructive pulmonary disease or asthma. Am Rev Respir Dis 1992;146:800-2.

4. Wilson AM, Blumsohn A, Jung RT, et al. Asthma and Cushing's syndrome. Chest 2000;117:593-4.

5. Do-Pham G, Pages C, Picard C, et al. A first case report of a patient with paraneoplastic dermatomyositis developing diffuse alveolar haemorrhage. Br J Dermatol 2010;163:227-8.

6. Fathi M, Dastmalchi M, Rasmussen E, et al. Interstitial lung disease, a common manifestation of newly diagnosed polymyositis and dermatomyositis. Ann Rheum Dis 2004;63:297-301.

7. Papadopoulou C, McCann LJ. The Vasculopathy of Juvenile Dermatomyositis. Front Pediatr 2018;6:284.

8. Sasaki H, Kohsaka H. Current diagnosis and treatment of polymyositis and dermatomyositis Mod Rheumatol 2018;28:913-21.

9. Minetto MA, D'Angelo V, Arvat E, et al. Diagnostic workup in steroid myopathy. Endocrine 2018;60:219-23.

Cite this article as: Fang Y, Yuan L, Zhao R, Jiang K, Dong X. Steroid myopathy in a child with diffuse alveolar hemorrhage: a case report. Ann Palliat Med 2021;10(6):7099-7106. doi: 10.21037/apm-21-816
10. Pereira RM, Freire de Carvalho J. Glucocorticoid-induced myopathy. Joint Bone Spine 2011;78:41-4.

11. Collard HR, Schwarz MI. Diffuse alveolar hemorrhage. Clin Chest Med 2004;25:583-92, vii.

12. Lara AR, Schwarz MI. Diffuse alveolar hemorrhage. Chest 2010;137:1164-71.

13. Susarla SC, Fan LL. Diffuse alveolar hemorrhage syndromes in children. Curr Opin Pediatr 2007;19:314-20.

14. Bowyer SL, LaMothe MP, Hollister JR. Steroid myopathy: incidence and detection in a population with asthma. $\mathrm{J}$ Allergy Clin Immunol 1985;76:234-42.

15. Kumar S. Steroid-induced myopathy following a single oral dose of prednisolone. Neurol India 2003;51:554-6.

16. Khan MA, Larson E. Acute myopathy secondary to oral steroid therapy in a 49-year-old man: a case report. J Med Case Rep 2011;5:82.

17. Kanda F, Okuda S, Matsushita T, et al. Steroid myopathy: pathogenesis and effects of growth hormone and insulinlike growth factor-I administration. Horm Res 2001;56 Suppl 1:24-8.

18. Dumitru D, Amato AA, Zwarts MJ. Electrodiagnostic medicine. 2nd ed. Philadelphia: Hanley \& Belfus, 2002. xi, $1524 \mathrm{p}$.

19. Huber AM. Juvenile Idiopathic Inflammatory Myopathies. Pediatr Clin North Am 2018;65:739-56.

20. Waldman R, DeWane ME, Lu J. Dermatomyositis: Diagnosis and treatment. J Am Acad Dermatol 2020;82:283-96. 